\title{
Masaoka-Koga Stage II
}

National Cancer Institute

\section{Source}

National Cancer Institute. Masaoka-Koga Stage II. NCI Thesaurus. Code C115033.

The tumor shows invasion into the capsule. 